\title{
BMJ Open Safety and efficacy of inpatient pulmonary rehabilitation for patients hospitalised with an acute exacerbation of chronic obstructive pulmonary disease: a systematic review protocol
}

\author{
Kai Zhu, ${ }^{1}$ Jagdeep Gill, ${ }^{1}$ Ashley Kirkham, ${ }^{2}$ Joel Chen, ${ }^{2,3}$ Amy Ellis, ${ }^{4}$ \\ Stephanie Crosbie, ${ }^{5}$ Holly Denson-Camp, ${ }^{2}$ Hannah Peters, ${ }^{1}$ Pat Camp (1) ${ }^{1}$
}

To cite: Zhu K, Gill J, Kirkham A, et al. Safety and efficacy of inpatient pulmonary rehabilitation for patients hospitalised with an acute exacerbation of chronic obstructive pulmonary disease: a systematic review protocol. BMJ Open 2021;11:e043377. doi:10.1136/ bmjopen-2020-043377

- Prepublication history and additional online supplemental material for this paper are available online. To view these files, please visit the journal online (http://dx.doi.org/10. 1136/bmjopen-2020-043377).

Received 17 September 2020 Accepted 26 May 2021

Check for updates

(C) Author(s) (or their employer(s)) 2021. Re-use permitted under CC BY-NC. No commercial re-use. See rights and permissions. Published by BMJ.

For numbered affiliations see end of article.

Correspondence to

Dr Pat Camp;

pat.camp@hli.ubc.ca

\section{ABSTRACT}

Introduction Pulmonary rehabilitation (PR) following an acute exacerbation of chronic obstructive pulmonary disease (AECOPD) reduces the risk of hospital admissions, and improves physical function and health-related quality of life. However, the safety and efficacy of in-hospital PR during the most acute phase of an AECOPD is not well established. This paper describes the protocol for a systematic review with meta-analysis to determine the safety and efficacy of inpatient acute care PR during the hospitalisation phase.

Methods and analysis Medical literature databases and registries MEDLINE, EMBASE, Physiotherapy Evidence Database, Cumulative Index to Nursing and Allied Health Literature, Canadian Agency for Drugs and Technologies in Health, CENTRAL, Allied and Complementary Medicine Database, WHO trials portal and ClinicalTrials.gov will be searched for articles from inception to June 2021 using a prespecified search strategy. We will identify randomised controlled trials that have a comparison of in-hospital PR with usual care. PR programmes had to commence during the hospitalisation and include a minimum of two sessions. Title and abstract followed by full-text screening will be conducted independently by two reviewers. A meta-analysis will be performed if there is sufficient homogeneity across selected studies or groups of studies. The Population, Intervention, Comparator, Outcomes and Study characteristics framework will be used to standardise the data collection process. The quality of the cumulative evidence will be assessed using the Grading of Recommendations, Assessment, Development and Evaluations framework.

Ethics and dissemination $A E C O P D$ results in physical limitations which are amenable to PR. This review will assess the safety and efficacy of in-hospital PR for AECOPD. The results will be presented in a peer-reviewed publication and at research conferences. Ethical review is not required for this study.

PROSPERO registration number CRD42021198877.

\section{INTRODUCTION}

Frequent acute exacerbations are a common feature of chronic obstructive pulmonary

\section{Strengths and limitations of this study}

- This will be the first study to evaluate the safety and efficacy of pulmonary rehabilitation (PR) specifically for the in-hospital period for patients admitted for an acute exacerbation of chronic obstructive pulmonary disease.

- This review benefits from a comprehensive search strategy across bibliographic databases, trial registries, scientific conferences and a grey literature database, ensuring the inclusion of as many relevant articles as possible.

- There may be studies that will be excluded that examine efficacy of inpatient PR but do not have a discharge measurement, which is required for this analysis.

disease (COPD), especially for patients with moderate to severe disease. ${ }^{1}$ Although most acute exacerbations of COPD (AECOPD) can be managed in the outpatient setting, many patients require hospital admission. The cost of these hospitalisations is immense, representing more than $25 \%$ of the total healthcare cost of COPD in the USA. $^{2}$ Decreases in quality of life (QoL), lung function, quadriceps muscle force and physical activity have been reported in patients during and after hospitalisation for AECOPD which, in many cases, may be permanent without intervention. ${ }^{3}$ Pitta et $a t^{4}$ reported that hospitalised patients with COPD show little improvement in their activity levels during their hospital stay, and by discharge only $9 \%$ of their day was spent standing or walking. Pulmonary rehabilitation (PR) during or shortly after an AECOPD may counteract these limitations in physical function. These programmes 
focus on mobility, muscle strength and/or exercise tolerance to mitigate the impact of the exacerbation and the hospitalisation period.

A 2016 Cochrane systematic review by Puhan et at examined the effects of 'early' PR (ie, during or shortly after an AECOPD) on future hospital admissions and other patient-related outcomes such as mortality, healthrelated QoL and exercise capacity in patients with COPD. However, this review included studies in which the PR intervention began in the hospital and was continued after discharge. The outcome measures assessed the effect of rehabilitation continued after discharge. It is possible that much of the benefit of PR during an AECOPD is seen during this post-discharge period. What is not clear is if a PR intervention delivered during the hospital admission is safe and results in changes in health status and physical function prior to discharge.

The Cochrane review authors concluded that early PR 'improves QoL and exercise capacity and is a safe intervention for patients with COPD after they have experienced an exacerbation,. ${ }^{5}$ Despite this, however, the 2017 American Thoracic Society/European Respiratory Society guideline for the management of AECOPD provided a conditional recommendation against the initiation of PR during hospitalisation. ${ }^{6}$ This caution was based on their own analysis that found that although PR initiated during hospitalisation improved exercise capacity, it also increased mortality. The increased mortality finding was strongly influenced by one study, ${ }^{7}$ which reported an increase in all-cause mortality in the intervention group. The excess mortality rate in the intervention group was detected at the 12-month postdischarge assessment, but did not occur during the hospitalisation, as there was no difference in hospital length of stay (LoS) or mortality between the groups during the hospital period. Nevertheless, the question remains regarding the safety of rehabilitation specifically during this acute care, inpatient phase, when patients are most ill with an AECOPD ${ }^{6}$ Clinicians who are considering implementing in-hospital PR for patients soon after admission must be confident when referring their patients to these programmes. Therefore, the purpose of this systematic review is to evaluate the safety and efficacy of in-hospital PR for patients admitted to hospital for an AECOPD.

\section{METHODS AND ANALYSIS}

This protocol has been prepared using the Preferred Reporting Items for Systematic Reviews and Meta-Analyses Protocols guidelines. ${ }^{8}$

\section{Types of studies}

We will select randomised controlled trials that have a comparison of in-hospital PR with usual care. We will include full-text, conference abstracts and unpublished data in any language.

\section{Types of participants}

This review will include studies of patients aged 19 years or older with a clinical diagnosis of COPD. The patient must be capable of physical activity (some form of movement whether it be active or stationary) and are hospitalised for an AECOPD at the time of the study. Any patients in an outpatient setting or in-patients admitted to hospital for any other reason besides their AECOPD are excluded from this study.

\section{Types of interventions}

The intervention of interest includes any rehabilitation programme that involves mobilisation, exercise or ambulation started while the patient is still hospitalised for an AECOPD. The rehabilitation programme must commence during the hospitalisation and include a minimum of two sessions. Rehabilitation programmes may include additional components such as selfmanagement education, psychological support, dietary advice and breathing exercises. We will exclude studies on PR programmes that included only neuromuscular stimulation or inspiratory muscle training but no physical exercise programme. Studies that include a subsequent outpatient rehabilitation programme following inpatient intervention are included only if there are preintervention and postintervention measurements for the duration of the hospitalisation. Any study that does not include information about how they measure/quantify patients' endpoints will be excluded.

\section{Outcome}

We will include studies that report measurable preintervention and postintervention data prior to discharge for one or more of the outcomes of interest. The primary outcomes are health-related QoL, functional exercise capacity and adverse events. The secondary outcomes are hospital readmissions within 30 days, and hospital LoS.

\section{Information sources}

Bibliographic databases MEDLINE, Embase, the Cumulative Index to Nursing and Allied Health Literature (CINAHL), the Physiotherapy Evidence Database (PEDro) and trial registries (Cochrane Controlled Register of Trials (CENTRAL), the US National Institutes of Health Ongoing Trials Register (ClinicalTrials. gov), the Allied and Complementary Medicine Database, PsycINFO and the WHO trials portal) will be searched up to June 2021 for potentially relevant articles using predefined search strategies (online supplemental appendix 1). Other searches will include the Canadian Agency for Drugs and Technologies in Health and handsearches of meeting abstracts from the American Thoracic Society and the European Respiratory Society scientific conferences. There will be no restriction on the language of the publication. A manual search of the reference lists of all included studies will be conducted to check for other possibly relevant articles. 


\section{Search strategy}

This review will use both keyword search terms as well as Medical Subject Headings terms in both MEDLINE and CENTRAL. In Embase we will use Emtree terms and in CINAHL we will use CINAHL headings. A key 'term' search strategy will be employed for the PEDro, ClinicalTrials.gov and WHO trials portal database. The proposed search strategies for MEDLINE is listed in online supplemental appendix 1 and will be adapted for use in the other databases. An academic librarian is a member of the study team and is involved in all aspects of developing the search strategies. The reference lists of all primary studies and review articles will be checked for additional references.

\section{Selection of studies}

The selection of studies for inclusion will be conducted in two stages. Two reviewers will independently screen the titles and abstracts of all potential studies identified in the search. The studies will be coded as 'yes' or 'no'. The full-text publications of included citations will then be screened by two reviewers according to our inclusion and exclusion criteria. Studies not in English will be translated using Google Translate. Any discrepancies at any stage will be resolved by the discussion and/or consultation with a third reviewer.

\section{Data collection process}

Two reviewers will independently collect information from all selected studies using a prespecified data extraction form. The form will be piloted on the first six selected studies and refined, as necessary. Any discrepancies will be resolved by discussion and/or consultation with a third reviewer. We will contact authors of eligible studies for any missing data. Using the Population, Intervention, Comparator, Outcomes, Study Characteristics framework, we will collect information on study design, interventions, participants, outcome measures and adverse events to systematise our data extraction (table 1).

\section{Data analysis and risk of bias}

Data will be synthesised by calculating mean differences and pooled ORs using random-effects models in Review Manager V.10. ${ }^{9}$ Heterogeneity will be assessed using forest plots, the $Q$ statistic and the $\mathrm{I}^{2}$ statistic. Heterogeneity will be examined using subgroup analysis and by analysing items from the quality assessment that indicate high risk versus low risk of bias (eg, blinding during data collection procedures). High heterogeneity will be considered present if $\mathrm{p}<0.1$ for the $\mathrm{Q}$ statistic or if $\mathrm{I}^{2}$ is $>50 \% .{ }^{10}$ If we find the included studies are sufficiently homogeneous in terms of design, study population and outcomes, we will conduct a meta-analysis to calculate a pooled effect estimate. If prohibited by heterogeneity, a narrative synthesis will be conducted instead.

Risk of bias assessment will be conducted independently by two reviewers on all studies using the criteria outlined in the Revised Cochrane Risk of Bias Tool for Randomised

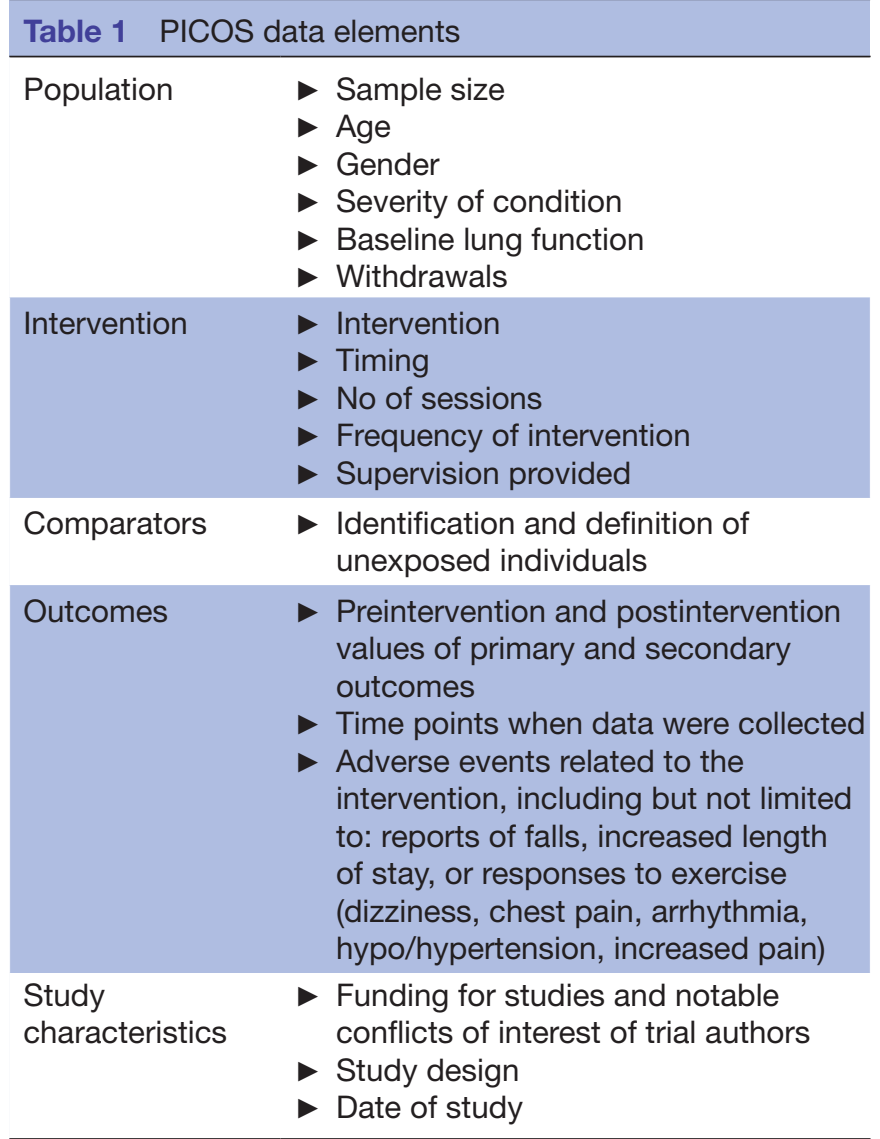

Trials. ${ }^{11}$ Any disagreements will be resolved by discussion and/or consultation with a third reviewer. To assess differences in health-related QoL, we will determine whether estimates and 95\% confidence limits between study groups exceed the minimal important difference (MID) for the Chronic Respiratory Questionnaire $( \pm 0.5$ points), ${ }^{12}$ the St George's Respiratory Questionnaire $( \pm 4$ points), ${ }^{13}$ the COPD Assessment Test (two points), ${ }^{14}$ the $6 \mathrm{~min}$ walk test $(\geq 30 \mathrm{~m})^{15}$ and the incremental shuttle walk test $(\geq 47.5 \mathrm{~m}) .{ }^{16}$ The unit of analysis is the participant. Patients who receive the usual care will be used as the control group in this study.

In order to account for selective reporting within studies, we will assess all available protocols for the included studies using the trial registries ClinicalTrials. gov and the WHO trials portal. Publication bias across studies will be assessed using funnel plots. The quality of the cumulative evidence for each outcome will be assessed using the Grading of Recommendations, Assessment, Development and Evaluations framework which includes the risk of bias, consistency, directness, precision and publication bias.

\section{Patient and public involvement}

There was no patient involved in the development of this protocol. 


\section{ETHICS AND DISSEMINATION}

Ethical review is not required as this study is a systematic review. It is our intention to submit the results of our review for peer-reviewed publication and to present our findings at scientific conferences.

\section{DISCUSSION}

PR plays an important role in the management of AECOPD. A large body of evidence shows that PR in patients with AECOPD helps to improve QoL, symptom management and disease prognosis, ${ }^{5}$; however, to our knowledge, there has been no review to assess the acute in-hospital use of PR for AECOPD. This review will determine the safety and efficacy of initiating physical rehabilitation, in patients with AECOPD, during their hospitalisation and will provide suggestions for future guidelines and recommendations.

\section{Author affiliations}

${ }^{1}$ Faculty of Medicine, The University of British Columbia, Vancouver, British Columbia, Canada

${ }^{2}$ Centre for Heart Lung Innovation, The University of British Columbia, Vancouver, British Columbia, Canada

${ }^{3}$ Respiratory Medicine, University of Calgary, Calgary, Alberta, Canada

${ }^{4}$ Surrey Memorial Hospital, Fraser Health Authority, Surrey, British Columbia, Canada

${ }^{5}$ S. Crosbie Consulting, Vancouver, British Columbia, Canada

Correction notice This article has been corrected since it was first published. The PROSPERO registration number has been added.

Twitter Pat Camp @UBCPulmRehabRes

Contributors KZ, JG and PC drafted the protocol and developed the study design and the data extraction form. KZ, JG, AK, JC, AE, SC, HD-C, HP and PC contributed to the development of the search strategies. All authors read and approved the final version of the manuscript for submission.

Funding PC holds grants from the Canadian Institutes of Health Research and from the Canadian Lung Association.

Competing interests None declared.

Patient consent for publication Not required.

Provenance and peer review Not commissioned; externally peer reviewed.

Supplemental material This content has been supplied by the author(s). It has not been vetted by BMJ Publishing Group Limited (BMJ) and may not have been peer-reviewed. Any opinions or recommendations discussed are solely those of the author(s) and are not endorsed by BMJ. BMJ disclaims all liability and responsibility arising from any reliance placed on the content. Where the content includes any translated material, BMJ does not warrant the accuracy and reliability of the translations (including but not limited to local regulations, clinical guidelines, terminology, drug names and drug dosages), and is not responsible for any error and/or omissions arising from translation and adaptation or otherwise.

Open access This is an open access article distributed in accordance with the Creative Commons Attribution Non Commercial (CC BY-NC 4.0) license, which permits others to distribute, remix, adapt, build upon this work non-commercially, and license their derivative works on different terms, provided the original work is properly cited, appropriate credit is given, any changes made indicated, and the use is non-commercial. See: http://creativecommons.org/licenses/by-nc/4.0/.

ORCID iD

Pat Camp http://orcid.org/0000-0002-9152-8251

\section{REFERENCES}

1 Global strategy for the diagnosis, management, and prevention of chronic obstructive pulmonary disease -2020 report. global initiative for chronic obstructive lung disease, 2020, 2021. Available: https:// goldcopd.org/wp-content/uploads/2019/12/GOLD-2020-FINAL-ver1. 2-03Dec19_WMV.pdf

2 National Heart, Lung and Blood Institute. Morbidity and mortality: 2012 chart book on cardiovascular, lung, and blood diseases. United States National Institute of Health, 2012.

3 Cote CG, Dordelly LJ, Celli BR. Impact of COPD exacerbations on patient-centered outcomes. Chest 2007;131:696-704.

4 Pitta F, Troosters T, Probst VS, et al. Physical activity and hospitalization for exacerbation of COPD. Chest 2006;129:536-44.

5 Puhan MA, Gimeno-Santos E, Cates CJ, et al. Pulmonary rehabilitation following exacerbations of chronic obstructive pulmonary disease. Cochrane Database Syst Rev 2016;12:CD005305.

6 Wedzicha JA, Miravitlles M, Hurst JR, et al. Management of COPD exacerbations: a European Respiratory Society/American Thoracic Society guideline. Eur Respir J 2017;49:1600791.

7 Greening NJ, Williams JEA, Hussain SF, et al. An early rehabilitation intervention to enhance recovery during hospital admission for an exacerbation of chronic respiratory disease: randomised controlled trial. BMJ 2014;349:g4315.

8 Shamseer L, Moher D, Clarke M, et al. Preferred reporting items for systematic review and meta-analysis protocols (PRISMA-P) 2015: elaboration and explanation. BMJ 2015;350:g7647-25.

9 The Cochrane Collaboration. Review Manager (RevMano [computer program]. Version 5.3. Copenhagen: The Nordic Cochrane Centre, 2014.

10 Higgins JPT, Thomas J, Chandler J, eds. Cochrane handbook for systematic reviews of interventions version 6.2. Cochrane, 2021. www.training.cochrane.org/handbook

11 Sterne JAC, Savović J, Page MJ, et al. Rob 2: a revised tool for assessing risk of bias in randomised trials. BMJ 2019;366:1489 8:14898.

12 Schünemann HJ, Puhan M, Goldstein R, et al. Measurement properties and interpretability of the chronic respiratory disease questionnaire (CRQ). COPD 2005;2:81-9.

13 Schünemann HJ, Griffith L, Jaeschke R, et al. Evaluation of the minimal important difference for the feeling thermometer and the St. George's respiratory questionnaire in patients with chronic airflow obstruction. J Clin Epidemiol 2003;56:1170-6.

14 Kon SSC, Canavan JL, Jones SE, et al. Minimum clinically important difference for the COPD assessment test: a prospective analysis. Lancet Respir Med 2014;2:195-203.

15 Holland AE, Spruit MA, Troosters T, et al. An official European respiratory Society/American thoracic Society technical standard: field walking tests in chronic respiratory disease. Eur Respir $J$ 2014;44:1428-46.

16 Singh SJ, Puhan MA, Andrianopoulos V, et al. An official systematic review of the European respiratory Society/American thoracic Society: measurement properties of field walking tests in chronic respiratory disease. Eur Respir J 2014;44:1447-78. 